\title{
Strahlentherapie beim NSCLC: relevante dosisabhängige Herzgefahr
}

\begin{abstract}
Daten zur Strahlentherapie von Mammakarzinom oder Hodgkin-Lymphom zeigen eine erhöhte Rate von Herzerkrankungen aufgrund der Mitbestrahlung des Herzens erst nach vielen Jahren. Wie sieht dies beim nichtkleinzelligen Lungenkarzinom (NSCLC) aus.
\end{abstract}

Patier atienten mit NSCLC haben eine schlechte Prognose. Sie sind im Mittel älter, meist Raucher und haben oft bereits ein vorgeschädigtes Herz, sodass eine Kardiotoxizität möglicherweise früh zum Tragen kommen könnte. Deshalb analysierten Onkologen von der University of North Carolina, Chapel Hill, USA, Daten der 127 von ihnen zwischen 1996 und 2009 in prospektive Dosisfindungsstudien der Radiation Therapy Oncology Group eingebrachten Patienten mit NSCLC des Stadiums III hinsichtlich symptomatischer kardialer Ereignisse. Die meisten Patienten (72 \%) waren mit 72 Gy bestrahlt worden. Bei der Bestimmung des kardialen Risikos berücksichtigten die Autoren koronare
Herzkrankheiten vor Therapie und das Risiko nach dem WHO/International Society of Hypertension-Score (ISHS).

Nach median 8,8 Jahren entwickelten 26 der überlebenden Patienten (23\%) ein oder mehrere Ereignisse, wobei die mediane Zeit bis zum ersten Ereignis bei 26 Monaten lag. 7 Patienten erlitten einen Perikarderguss, 5 eine Herzinfarkt, 3 eine instabile Angina pectoris, 2 eine Perikarditis, 12 Herzrhythmusstörungen und einer eine Herzinsuffizienz.

In der univariaten Analyse waren die mittlere Herz-Strahlendosis (Hazard Ratio [HR] 1,03/Gy; $p=0,002$ ), eine vorbestehende koronare Herzerkrankung $(\mathrm{p}<001)$ und der ISHS $(\mathrm{p}=0,04) \mathrm{mit}$ späteren kardialen Ereignissen assozi- iert. Berücksichtigte man das Basisrisiko für eine Herzerkrankung, blieb als signifikanter Faktor nur die Herzdosis übrig. Die risikoadjustierten Ereignisraten lagen bei einer Herzdosis $<10$ Gy bei $4 \%$, bei $10-20$ Gy bei $7 \%$ und bei $>20$ Gy bei $21 \%$. Mit dem Gesamtüberleben waren die Herzdosen nicht assoziiert.

Fazit: Kardiale Ereignisse betrafen fast ein Viertel der mit hoher Dosis thorakal bestrahlten Patienten. Unabhängige Einflussfaktoren waren Herzdosis und vorbestehendes kardiales Risiko. Die Autoren sehen die Vermutung bestätigt, dass eine kardiale Toxizität beim NSCLC im Stadium III früh auftritt, damit relevant ist und man die Herzdosis deshalb möglichst klein halten sollte. Friederike Klein

Wang K et al. Cardiac Toxicity After Radiotherapy for Stage III Non-Small-Cell Lung Cancer: Pooled Analysis of Dose-Escalation Trials Delivering 70 to $90 \mathrm{~Gy}$. J Clin Oncol. 2017;35(13):1387-94.

\section{NSCLC: Kombiniert gegen Hirnmetastasen}

Bei Hirnmetastasen eines nichtkleinzelligen Lungenkarzinoms (NSCLC) mit EGFR-Mutation kommen als Therapieoptionen die stereotaktische Strahlenchirurgie (SRS), die Ganzhirnbestrahlung (WBRT) und die EGFR-Tyrosinkinaseinhibition (TKI) infrage. Unklar ist das optimale Management.

$\mathrm{R}_{\mathrm{t}}^{\mathrm{s}}$ etrospetiv wurden daher 351 Patienten mit EGFR-mutiertem NSCLC und Hirnmetastasen untersuchten, die zwischen 2008 und 2014 in 6 Institutionen entweder zunächst eine SRS, dann einen EGFR-TKI erhalten hatten (SRSKohorte) oder erst eine WBRT und dann einen EGFR-TKI (WBRT-Kohorte) oder erst einen EGFR-TKI und dann eine der beiden strahlentherapeutischen Therapien (EGFR-TKI-Kohorte). Ausgeschlossen waren Patienten, die bereits vor Entwicklung der Hirnmetastasen EGFRTKI erhalten hatten oder eine EGFRTKI-Resistenzmutation aufwiesen.

Das mediane Gesamtüberleben (OS) nach Diagnose der Hirnmetastasen lag in der SRS-Kohorte $(n=100)$ bei $46 \mathrm{Mo-}$ naten, in der WBRT-Kohorte $(\mathrm{n}=120)$ bei 30 Monaten und in der EGFR-TKIKohorte $(\mathrm{n}=131)$ bei 25 Monaten.

Der Unterschied zwischen SRS und EGFR-TKI als primäre Therapie bei Hirnmetastasen eines NSCLC war signifikant $(\mathrm{p}<0,001)$. Auch in einer multivariaten Analyse war die SRS der EGFR-TKI-Therapie beim OS überlegen. Außerdem war eine WBRT gegenüber einer EGFR-TKI-Therapie mit einem längeren OS assoziiert. Dabei waren SRSund EGFR-TKI-Kohorten bezüglich prognostischer Faktoren weitgehend ähnlich, während die WBRT-Kohorte mit höherer Wahrscheinlichkeit eine ungünstige Prognose aufwies ( $\mathrm{p}=0,001)$. Weitere Einflussfaktoren für ein längeres OS waren neben der therapeutischen Strategie ein jüngeres Alter, ein guter
Performancestatus, eine Mutation im Exon 19 des EGFR-Gens und das Fehlen von extrakraniellen Metastasen.

Fazit: Nach der retrospektiven Auswertung geht die primäre EGFR-TKI-Therapie mit verzögerter Bestrahlung bei intrakranieller Metastasierung eines EGFR-mutierten NSCLC mit einem schlechteren OS einher als eine SRS mit nachfolgender EGFR-TKI-Behandlung, die zudem mögliche neurokognitive Einschränkungen durch eine WBRT vermeiden kann. Es ist dringend eine prospektive Studie zu fordern, die randomisiert die Sequenz SRS - EGFR-TKI und EGFR-TKI - SRS bei intrakraniellem Progress eines EGFR-mutierten NSCLC untersucht. Bis dahin sollte nach Meinung der Forscher upfront die SRS erfolgen.

Friederike Klein

Magnuson WJ et al. Management of Brain Metastases in Tyrosine Kinase Inhibitor-Naïve Epidermal Growth Factor Receptor-Mutant Non-Small-Cell Lung Cancer: A Retrospective Multi-Institutional Analysis. J Clin Oncol. 2017;35(10):1070-7. 